Non-invasive brain stimulation for stroke recovery - ready for the big time?

\title{
Nick S Ward
}

Sobell Department of Motor Neuroscience

UCL Institute of Neurology

Queen Square

London WC1N 3BG

Email: n.ward@ucl.ac.uk

Tel: 02034488762

Twitter: @dr_nickward

Word count: 886 
Stroke is the commonest cause of physical disability in the world and yet we are still struggling for consensus on how best to treat stroke survivors in order to maximize recovery after the acute event.

When it comes to treating motor impairment there are two complementary therapeutic approaches to consider. Firstly, physical therapies which are based on massed practice followed by incorporation of improvements into functional tasks through the instruction and knowledge provided by skilled physiotherapists and occupational therapists (1). Secondly, there are a number of experimental approaches under investigation which aim to increase the effects of training by enhancing the potential for use-dependent plasticity. These 'primers' of the motor system might include drugs (e.g. fluoxetine(2)), specific forms of activity (activepassive bilateral arm training (3), aerobic exercise (4)) and non-invasive brain stimulation, (e.g. transcranial direct current stimulation, tDCS $(5,6)$ ). None of these interventions should be thought of as treatments, but rather as tools for enhancing the effects of conventional practice-based treatment, which in the case of post-stroke motor impairment is currently physical therapy.

It is worth stating at this point that the amount of physical therapy currently offered for upper limb impairment is likely to be far too low (7). Pragmatic attempts to increase the dose (here referring to time) are not aspirational and appear not to alter longer term motor outcomes. Unresolved issues in post-stroke motor rehabilitation are therefore (i) how do we deliver higher doses of appropriate physical therapy (whether in the early or chronic phase post-stroke) in a motivating environment and (ii) is it possible to maximise the effects and retention of physical therapy by enhancing use dependent plasticity?

TDCS involves placing electrodes on the scalp in a particular montage in order to generate constant low levels of electrical current flow through the brain. It is often claimed that this either increases or decreases the neuronal excitability in specific brain areas being stimulated based on the placement of the electrodes and which type of stimulation is being used, but the complexity of the variables involved tell us this is unlikely to be the case (8). At present, it is probably safe to say that tDCS can change some aspect of brain neurophysiology as demonstrated in human and animal studies, but exactly what it is doing in individuals is unclear.

In this issue of JNNP, Kang et al., (5) perform a systematic review and metaanalysis to address whether using tDCS before or during motor training not only increases motor performance but also improves retention of new motor skills. For anyone embarking on a study of the effects of tDCS in stroke rehabilitation there are an intimidating range of decisions to make; (i) should 'excitatory' anodal tDCS, 'inhibitory' cathodal tDCS, or bihemispheric tDCS be used; (ii) should the ipsilesional or contralesional sensorimotor cortex be targeted; (iii) is tDCS more effective in the early or chronic phase post-stroke; (iv) should tDCS be applied before or during training; (v) what sort of training should be used; (vi) does any of this matter? There have been recent criticisms of the selective nature of metaanalyses in this field (9), but here Kang et al., have included a wide range of publications in order to overcome within-study biases or idiosyncrasies. In theory at least, this should provide some answers to the key questions.

Seventeen studies were examined and the results are instructive for the field of neurorehabilitation neuroscience for a number of reasons. Firstly, the meta- 
analysis was positive. Overall, there was an effect size of 0.59 in favour of tDCS improving the effects of physical therapy when measured at least 5 days after the intervention. This effect size is in keeping with those found by other metaanalyses of non-invasive brain stimulation in stroke $(10,11)$. However, the questions about how, where, and when remain unresolved. Based on the studies available, it does not seem to matter whether excitatory or inhibitory tDCS is applied to the ipsilesional or contralesional hemisphere respectively. Nor does it seem important whether it is given early or late after stroke, before or after training, or even what sort of training is provided. There is no difference in any comparison because everything seems to work to the same degree.

So what do we do with this information? It is tempting to take this relatively nonspecific positive result as a signal to start phase 3 randomised clinical trials. If all combinations seem to work, why not apply it in a broad brush approach? Perhaps tDCS has a non-specific effect and it could work in a heterogeneous condition such as stroke. Crucially however, the known variability of its effect (12) points to a quite specific mechanism, it's just that we don't know what it is yet. Now is the time to step back and ask what it is we think we are doing here. How should tDCS be delivered and who is most likely to benefit? If, for example, we believe that it is delivering a known amplitude of current flow to a specific brain region is the key, then we would be better off asking whether we are actually achieving this rather than pressing on with a 'black box' approach. You never know, we might achieve an impressive effect size that will change practice if we understand how to use it. It's not time to stop asking questions yet.

\section{References}

1. Ward NS, Kelly K, Brander F. The future of stroke rehabilitation: upper limb recovery $A C N R$ 2015;15(4):6-8.

2. Chollet F, Tardy J, Albucher J-F, et al. Fluoxetine for motor recovery after acute ischaemic stroke (FLAME): a randomised placebo-controlled trial. Lancet Neurol. 2011;10:123-30.

3. Stinear CM, Petoe MA, Anwar S, et al. Bilateral priming accelerates recovery of upper limb function after stroke: a randomized controlled trial. Stroke. 2014;45:205-10.

4. Mang CS, Campbell KL, Ross CJD, et al. Promoting neuroplasticity for motor rehabilitation after stroke: considering the effects of aerobic exercise and genetic variation on brain-derived neurotrophic factor. Phys Ther. 2013;93:1707-16.

5. Kang N, Summers JJ, Cauraugh JH. Transcranial direct current stimulation facilitates motor learning post-stroke: a systematic review and metaanalysis. J Neurol Neurosurg Psychiatry. 2015 Aug 28; [Epub ahead of print]

6. Monti A, Ferrucci R, Fumagalli M, Mameli F, Cogiamanian F, Ardolino G, et al. Transcranial direct current stimulation (tDCS) and language. J Neurol Neurosurg Psychiatry. 2013;84:832-42. 
7. Lohse KR, Lang CE, Boyd LA. Is more better? Using metadata to explore doseresponse relationships in stroke rehabilitation. Stroke. 2014;45:2053-8.

8. de Berker AO, Bikson M, Bestmann S. Predicting the behavioral impact of transcranial direct current stimulation: issues and limitations. Front Hum Neurosci. 2013;7:613.

9. Nitsche MA, Bikson M, Bestmann S. On the Use of Meta-analysis in Neuromodulatory Non-invasive Brain Stimulation. Brain Stimulat. 2015;8:666-7.

10. Butler AJ, Shuster M, O'Hara E, et al. A meta-analysis of the efficacy of anodal transcranial direct current stimulation for upper limb motor recovery in stroke survivors. J Hand Ther. 2013;26:162-70.

11. Hsu $\mathrm{W}-\mathrm{Y}$, Cheng $\mathrm{C}-\mathrm{H}$, Liao $\mathrm{K}-\mathrm{K}$, et al. Effects of repetitive transcranial magnetic stimulation on motor functions in patients with stroke: a metaanalysis. Stroke. 2012;43:1849-57.

12. Wiethoff S, Hamada M, Rothwell JC. Variability in response to transcranial direct current stimulation of the motor cortex. Brain Stimulat. 2014;7:46875. 